\section{Case Reports in Ophthalmology}

\title{
Optical Coherence Tomography Angiography: Employing a Novel Technique for Investigation in Vogt-Koyanagi-Harada Disease
}

\author{
Panagiotis Giannakouras $^{\mathrm{a}} \quad$ Konstantinos Andreanos $^{\mathrm{a}} \quad$ Barbara Giavi $^{\mathrm{a}}$ \\ Andreas Diagourtas ${ }^{a}$ \\ ${ }^{a}$ Athens University Eye Clinic, G. Gennimatas General Hospital, Athens, Greece
}

\section{Keywords}

Optical coherence tomography angiography - Superficial and deep retinal plexus .

Choriocapillaris · Vogt-Koyanagi-Harada disease

\begin{abstract}
Purpose: To report a case of Vogt-Koyanagi-Harada (VKH) disease and describe the imaging findings by means of optical coherence tomography angiography (OCTA). Methods: Medical and ophthalmological history, ophthalmological examination, laboratory evaluation, B-scan ultrasonography, fluorescein and indocyanine angiography, and optical coherence tomography (OCT) were performed at baseline, as well as OCTA. Results: A 50-year-old healthy female presented with decreased vision in both eyes. A Topcon DRI OCT Triton Plus swept source OCT system was used to visualize and evaluate the retinal and choroidal vascular plexus. Patchy and confluent dark areas in the superficial and deep retinal capillary plexus and choriocapillaris corresponded to areas of hypoperfusion, analyzed as areas of ischemia. Conclusions and Importance: VKH disease is characterized by ocular, neurological, and integumentary findings in its complete form. We present a case of incomplete disease in a 50-year-old female evaluated by means of OCTA which is a novel technique that provides depth-resolved images of the retina and choroidal microvasculature without dye injection that allows better visualization and detailed evaluation of the retinal and choroidal vascular plexus.

(c) 2017 The Author(s)
\end{abstract}




\section{Introduction}

Vogt-Koyanagi-Harada (VKH) disease is an autoimmune granulomatous inflammatory systemic disorder presumed to occur due to erroneous autoimmune sensitization of T lymphocytes against melanocytes in genetically susceptible individuals [1]. It is associated with HLA-DR4, notably HLA-DRB1*0405, which recognizes various melanocyte proteins. It is characterized by ocular, neurological, and integumentary manifestations in its complete form [2]. The ophthalmological signs include bilateral granulomatous uveitis with mild vitritis, yellow-white retinal exudates in the retinal pigment epithelium, and multifocal serous retinal detachments [3]. Dalen-Fuchs nodules (peripheral yellow-white choroidal granulomata) may be apparent. Optic disc swelling is a frequent finding. The inflammation primarily involves the choroidal stroma with subsequent involvement of the retinal pigment epithelium and outer retina [4]. Upon resolution of the acute uveitic phase, patients enter the convalescent phase characterized by choroidal depigmentation, depigmented areas at the limbus (called Sugiura's sign), vitiligo, poliosis, and alopecia. Episodes of recurrent anterior uveitis can occur in up to $50 \%$ of cases. VKH disease accounts for $6-8 \%$ of all uveitis in Asia, 1-2\% in the Middle East, 1-4\% in North America, and 2-4\% in Brazil [5]. We report a case of incomplete VKH disease starting as bilateral papillitis. We fully investigated the case with laboratory evaluation, B-scan ultrasonography, magnetic resonance imaging (MRI), fluorescein and indocyanine green angiography (FA, ICGA), and optical coherence tomography (OCT). OCT angiography (OCTA) is a novel technique, which provides mapping of the choroidoretinal plexus without the need for a dye injection. This case report provides an attempt to analyze the results observed in the choroidal plexus using OCTA.

\section{Case Report}

We report a case of VKH disease and describe the imaging findings by means of OCTA. A 50-year-old Caucasian female was referred to the ophthalmic emergency department when diagnosed with bilateral optic disc swelling from a neurological clinic. She denied any focal neurological condition but she stated that she experienced ophthalmalgia initially in her right eye and then in her left, progressing the last month. She reported contact with cats and ischialgia after fatigue. Furthermore, she had no remarkable past medical and ophthalmological history. Upon examination, the best corrected visual acuity was $0.2 \operatorname{logMAR}$ in both eyes with relative afferent pupillary defect in the right eye. Slit lamp examination showed 2+ cells and $3+$ flare in the anterior chamber of each eye with normal intraocular pressure as well as clear lenses and cornea without any precipitates. Iridocapsular synechiae were noted on the right eye. The Isihara test was normal for both eyes. B-scan ultrasound biomicroscopy revealed diffuse thickening of the choroid with low to medium reflectivity. On Humphrey automated perimetry, blind spots were enlarged in both eyes. Dilated fundus and enhanced depth imaging OCT examination revealed bilateral optic disc swelling and posterior pole serous retinal detachment. Spectral domain OCT revealed subretinal fluid and fibrin deposition. The choroid appeared thickened (Fig. 1). FA and ICGA were performed using spectral domain OCT (Spectralis; Heidelberg Engineering, Heidelberg, Germany). FA, late phase, showed bilateral optic disc swelling with staining of the optic discs, and ICGA depicted patchy hyperfluorescent areas from the early mid-phase and dots in the late phase without 


\section{Case Reports in Ophthalmology}

Case Rep Ophthalmol 2017;8:362-369

DOI: $10.1159 / 000477611$

(c) 2017 The Author(s). Published by S. Karger AG, Basel www.karger.com/cop

Giannakouras et al.: Optical Coherence Tomography Angiography: Employing a Novel Technique for Investigation in Vogt-Koyanagi-Harada Disease

pigment epithelial detachment (Fig. 2). Another feature studied was the presence of some peripapillary pinpoints, with some pinpoints in the macular area as well (Fig. 2). This finding serves as a useful prognostic sign for the evolution of treatment as it indicates that the disease is in the acute phase. The en face and cross-sectional OCTA images were analyzed to study various retinochoroidal layers. The superficial and deep retinal capillary plexus did not show any pathological changes at presentation. OCTA showed round hyporeflective areas bilaterally, which represent areas of choriocapillaris hypoperfusion. On OCTA the choriocapillaris layer showed dark patchy areas corresponding to ischemia, whereas superficial and deep retinal capillary plexus showed a decreased foveal avascular zone (Fig. 3). On corresponding structural en face and cross-sectional OCT B-scans, there was no evidence of signal loss, which suggests that the hyporeflective areas likely confirm the hypoperfusion. Furthermore, clinical examination revealed a systemic blood pressure of 125/85 mm Hg. Neurological and rheumatological examination was nonrevealing. Anti-thyroid peroxidase antibody and anti-thyroid globulin antibodies were found to be above normal, whereas the rest of the hematological laboratory investigations revealed normal results. Markers of autoimmunity revealed HLA-DR4 antigen. Serological testing for the diagnosis of infectious pathologies was negative. Thin section brain MRI revealed normal findings. Lumbar puncture revealed cerebrospinal fluid pressure of $22 \mathrm{~cm} \mathrm{H}_{2} \mathrm{O}$ and microscopically lymphocytic pleocytosis. The patient was prescribed with oral methylprednisolone $64 \mathrm{mg}$ once daily and topical steroids initially every hour and then on a tapering schedule after 2 weeks, with omeprazole $20 \mathrm{mg}$ and a salt restriction diet. Two weeks after methylprednisolone therapy the patient had a significant recovery with no cells or flare in the anterior chamber of both eyes, optic disc swelling improvement, and visual acuity of $0.04 \log$ MAR bilaterally.

\section{Discussion}

Current diagnostic criteria for VKH disease require the presence of bilateral ocular involvement. FA shows hyperfluorescent dots, which progressively become more hyperfluorescent as dye pools into the subretinal space and optic nerve staining [6, 7]. FA is essential in differentiating between the different diagnostic possibilities. Subretinal fluid, pigment epithelial detachment, and subretinal septa can all be visualized in OCT investigating VKH disease. Several studies agree that FA findings together with ICGA are especially useful during follow-up to detect persistence of disease $[8,9]$. ICGA is a very sensitive test in the detection of very small inflammatory changes in the choriocapillaris and choroidal stromal vessels $[10,11]$. Nevertheless, it is an invasive imaging examination with the potential risk of dyerelated adverse effects. In this case report, we studied an incomplete VKH disease in a 50year-old female. A high-definition swept source OCT B-scan, a full-color fundus photograph, and a swept source OCTA (Topcon DRI OCT Triton Plus) were performed. OCTA is a novel technique that provides depth-resolved images of the retina and choroidal microvasculature without dye injection $[12,13]$. All scans were acquired over a $3 \times 3 \mathrm{~mm}$ field of view in about $3 \mathrm{~s}$ of total OCT scan time. The analysis of the choriocapillaris layer showed dark patchy areas corresponding to ischemia. Thus, hypoperfusion (loss of choriocapillaris) found on OCTA correlates well with findings from OCT and ICGA (Fig. 3). The superficial and deep retinal capillary plexus, particularly in the right eye, showed a decrease in foveal avascular zone. Regarding the deep capillary plexus, we noticed a pattern of concentric cycles in both eyes, corresponding to disruption of the outer plexiform layer due to intrusion of the outer nuclear layer (Fig. 4). The same pattern was observed in our department in 2 cases of impending 
retinal detachment. The subfoveal choroidal thickness was $406 \mu \mathrm{m}$ at presentation in the right eye, which was reduced to $319 \mu \mathrm{m}$ at the follow-up visit (Fig. 3). OCTA when combined with other imaging systems is effective in highlighting the pathological changes in vascular diseases and in ocular inflammatory diseases, such as VKH. In combination with enhanced depth imaging OCT, OCTA can be used to follow evolution in vascular and inflammatory disorders at frequent intervals without any side effects for the patient. It can be safely and easily repeated and allows frequent follow-up imaging. OCTA greatly supplements the technique of ICGA and may reduce its need in routine clinical practice [4]. However, hitherto OCTA allows assessment only of the macular area and does not show leakage, pooling, or staining. In OCTA images hemorrhages hide the capillaries, and exudates cannot be assessed efficiently. In ischemic fields capillaries dissipate. OCTA provides poor visualization of the choroidal vascular plexus of a thickened and inflamed choroid. This case highlights the potential utility of OCTA, a novel technique without the potential risk of dye-related adverse effects, which confirms the results of the other imaging investigations with the advantage of fine evaluation of the retinal and choroidal vascular plexus. Imaging plays an important role in the diagnosis and management of patients with VKH. In our case report OCTA appeared to be an effective tool in detecting the presence of disease activity in VKH disease. Further research may confirm our observation.

\section{Conclusion}

Imaging is decisive in the management of patients with VKH. OCTA can enhance the illustration and assist in a detailed evaluation of the choroidal plexus. In our case report OCTA appeared to be a useful tool in detecting the presence of disease activity in VKH disease. OCTA can provide the clinician with an efficient adjunct that will help him determine the disease activity. Further research may confirm our observation.

\section{Statement of Ethics}

The authors have no ethical conflicts to disclose.

\section{Disclosure Statement}

The authors have no proprietary interests, financial support, or any other conflicts of interest to report.

\section{References}

1 Da Silva FT, et al: Enhanced depth imaging optical coherence tomography in long-standing VogtKoyanagi-Harada disease. Br J Ophthalmol 2013;97:70-74.

-2 Read RW, Holland GN, Rao NA, et al: Revised diagnostic criteria for Vogt-Koyanagi-Harada disease: report of an international committee on nomenclature. Am J Ophthalmol 2001;131:647-652.

-3 Yamaguchi Y, Otani T, Kishi S: Tomographic features of serous retinal detachment with multilobular dye pooling in acute Vogt-Koyanagi-Harada disease. Am J Ophthalmol 2007;144:260-265.

4 Kanika Agarwal, et al: The role of optical coherence tomography angiography in the diagnosis and management of acute Vogt-Koyanagi-Harada disease. Ocul Immunol Inflamm 2016;2016:1-12 


\section{Case Reports in Ophthalmology}

\begin{tabular}{l|l}
\hline Case Rep Ophthalmol 2017;8:362-369 \\
\hline DOI: 10.1159/000477611 & $\begin{array}{l}\text { @ 2017 The Author(s). Published by S. Karger AG, Basel } \\
\text { www.karger.com/cop }\end{array}$ \\
\hline
\end{tabular}

Giannakouras et al:: Optical Coherence Tomography Angiography: Employing a Nove Technique for Investigation in Vogt-Koyanagi-Harada Disease

5 Sakata VM, et al: Diagnosis and classification of Vogt-Koyanagi-Harada disease. Autoimmun Rev 2014;13:550-555.

-6 Fardeau C, Tran TH, Gharbi B, Cassoux N, Bodaghi B, LeHoang P: Retinal Fluorescein and indocyanine green angiography and optical coherence tomography in successive stages of Vogt-Koyanagi-Harada disease. Int Ophthalmol 2007;27:163-172.

7 Jap A, Chee SP: Imaging in the diagnosis and management of Vogt-Koyanagi-Harada disease. Int Ophthalmol Clin 2012;52:163-172.

8 Chee SP, Jap A, Cheung CM: The prognostic value of angiography in Vogt-Koyanagi-Harada disease. Am J Ophthalmol 2010;150:888-893.

-9 Herbort CP, Mantovani A, Bouchenaki N: Indocyanine green angiography in Vogt-Koyanagi-Harada disease: angiographic signs and utility in patient follow-up. Int Ophthalmol 2007;27:173-182.

10 Khairallah M, Ben Yahia S, Attia S, Zaouali S, Jelliti B, Jenzri S, Ladjimi A, Messaoud R: Indocyanine green angiographic features in multifocal chorioretinitis associated with West Nile virus infection. Retina 2006;26:358-359.

11 Machida S, Tanaka M, Murai K, Takahashi T, Tazawa Y: Choroidal circulatory disturbance in ocular sarcoidosis without the appearance of retinal lesions or loss of visual function. Jpn J Ophthalmol 2004;48:392-396.

-12 Jia Y, Tan O, Tokayer J, Potsaid B, Wang Y, Liu JJ, Kraus MF, Subhash H, Fujimoto JG, Hornegger J, Huang D: Split-spectrum amplitude-decorrelation angiography with optical coherence tomography. Opt Express 2012;20:4710-4725.

13 Makita S, Jaillon F, Yamanari M, Miura M, Yasuno Y: Comprehensive in vivo micro-vascular imaging of the human eye by dual-beam-scan Doppler optical coherence angiography. Opt Express 2011;19:12711283.
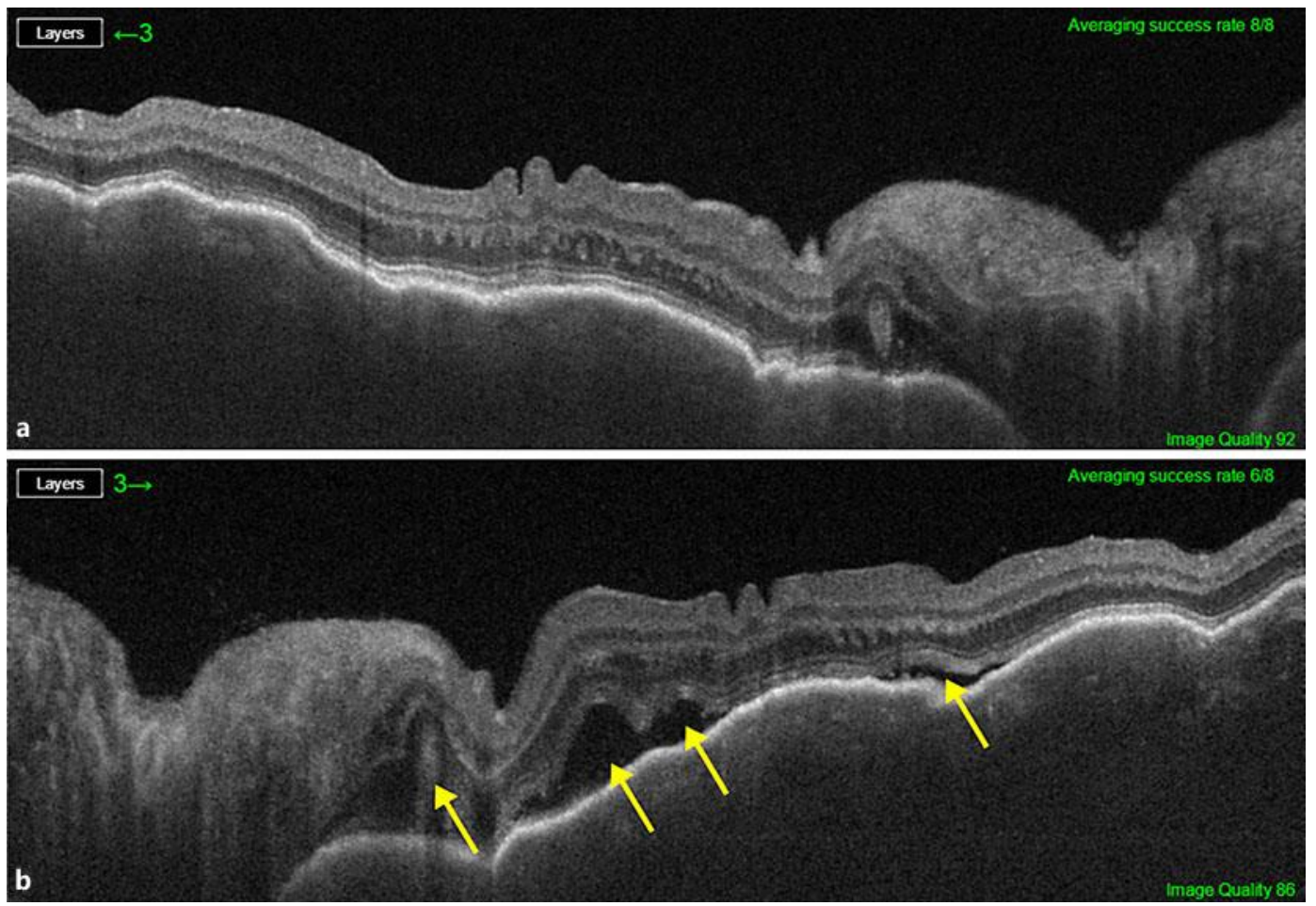

Fig. 1. a Enhanced depth imaging OCT through the macular detachment in a 50-year-old female with probable Vogt-Koyanagi-Harada disease shows subretinal fluid with membrane structure, irregular undulated retinal pigment epithelial lines due to choroidal folds, and edema. b OCT scan in the same patient shows subretinal fluid with fibrin deposition. The choroid appears thickened. 


\section{Case Reports in Ophthalmology}

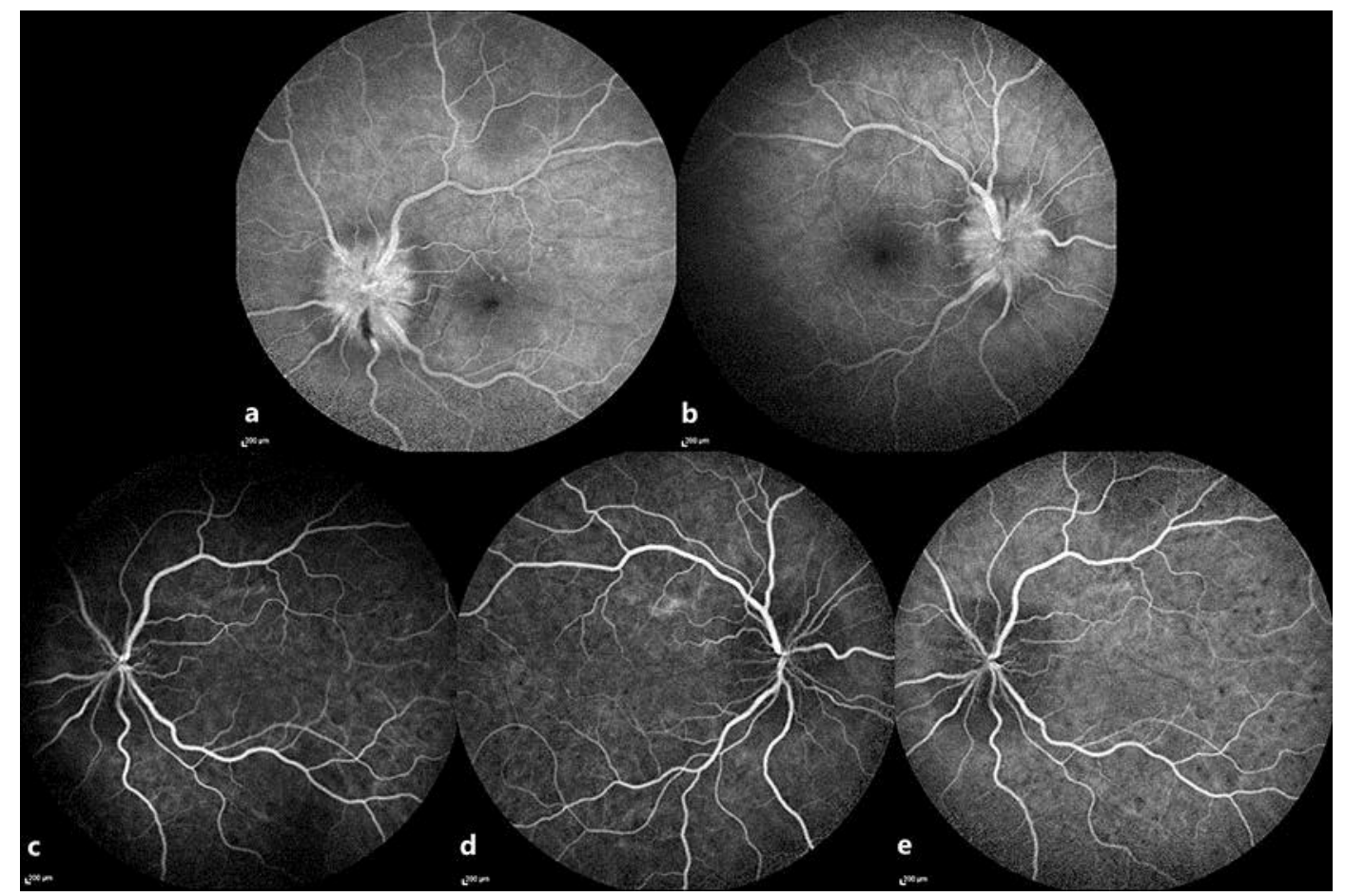

Fig. 2. a, b Late stage fluorescein angiography shows optic nerve edema with retinal vascular engorgement from inflammatory induced stasis. c-e Indocyanine green angiography of the same patient showing patchy hyperfluorescent areas in the early mid-phase and hypofluorecent dots in the late phase without pigment epithelial detachment.

Giannakouras et al: Optical Coherence Tomography Angiography: Employing a Novel Technique for Investigation in Vogt-Koyanagi-Harada Disease 


\section{Case Reports in Ophthalmology}

\begin{tabular}{l|l}
\hline Case Rep Ophthalmol 2017;8:362-369 \\
\hline DOI: 10.1159/000477611 & $\begin{array}{l}\text { ○ 2017 The Author(s). Published by S. Karger AG, Basel } \\
\text { www.karger.com/cop }\end{array}$ \\
\hline
\end{tabular}

Giannakouras et al.: Optical Coherence Tomography Angiography: Employing a Nove Technique for Investigation in Vogt-Koyanagi-Harada Disease

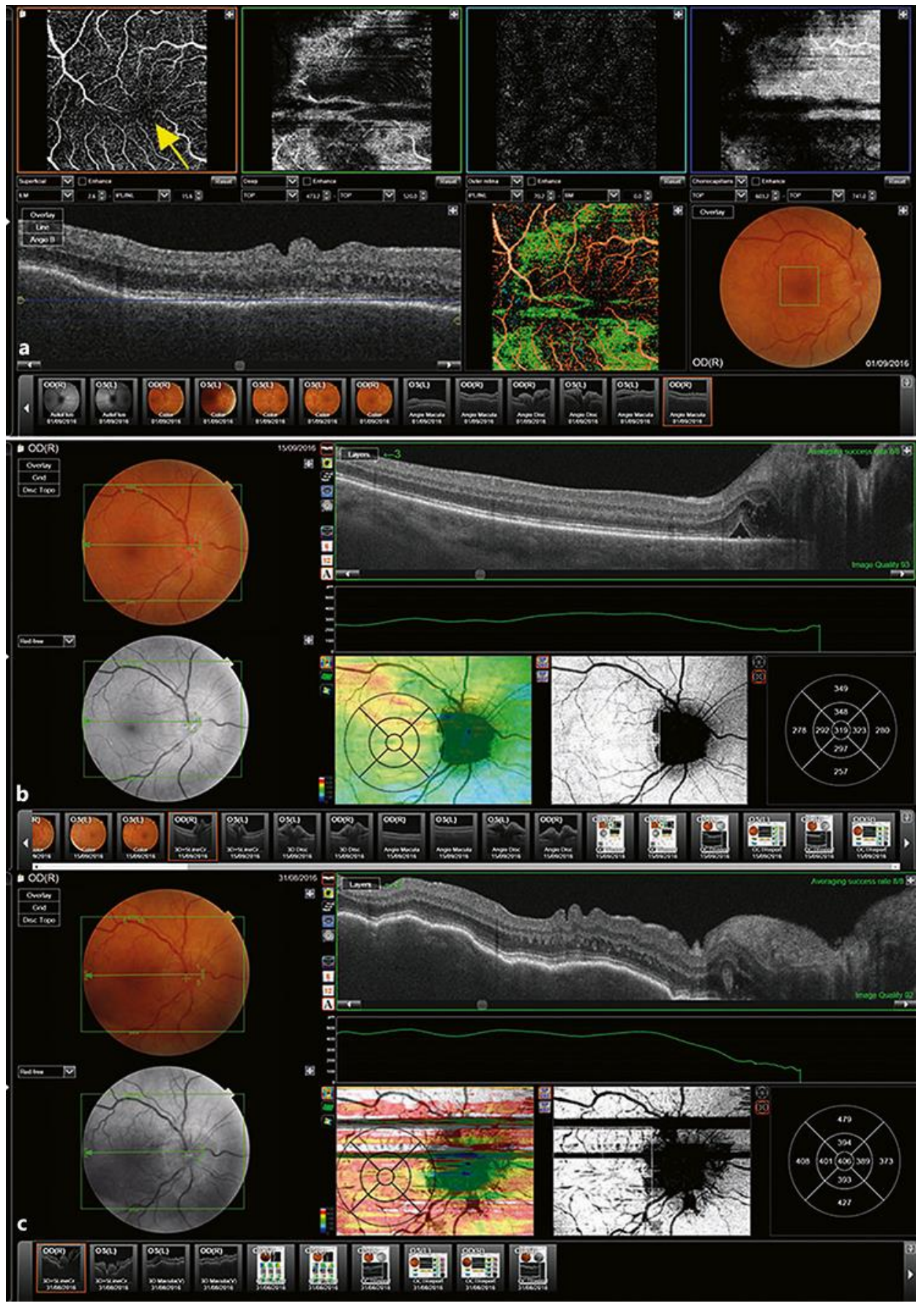

Fig. 3. Topcon DRI OCT Triton Plus swept source OCT system. The fundus photograph of both eyes reveals optic disc edema along with exudative posterior pole detachment. a OCT angiography (upper row images) shows areas of microvascular infarction at the level of the choroidal plexus. The superficial capillary plexus shows a decreased foveal avascular zone (arrow). At the level of the deep capillary plexus we notice a pattern of concentric cycles corresponding to disruption of the outer plexiform layer due to intrusion of the outer nuclear layer. b, c The subfoveal thickness at presentation and at the follow-up visit was 406 and $319 \mu \mathrm{m}$, respectively, in the right eye. 
Case Reports in
Ophthalmology

Case Rep Ophthalmol 2017;8:362-369 DOI: 10.1159/000477611

c 2017 The Author(s). Published by S. Karger AG, Basel www.karger.com/cop

Giannakouras et al:: Optical Coherence Tomography Angiography: Employing a Novel Technique for Investigation in Vogt-Koyanagi-Harada Disease
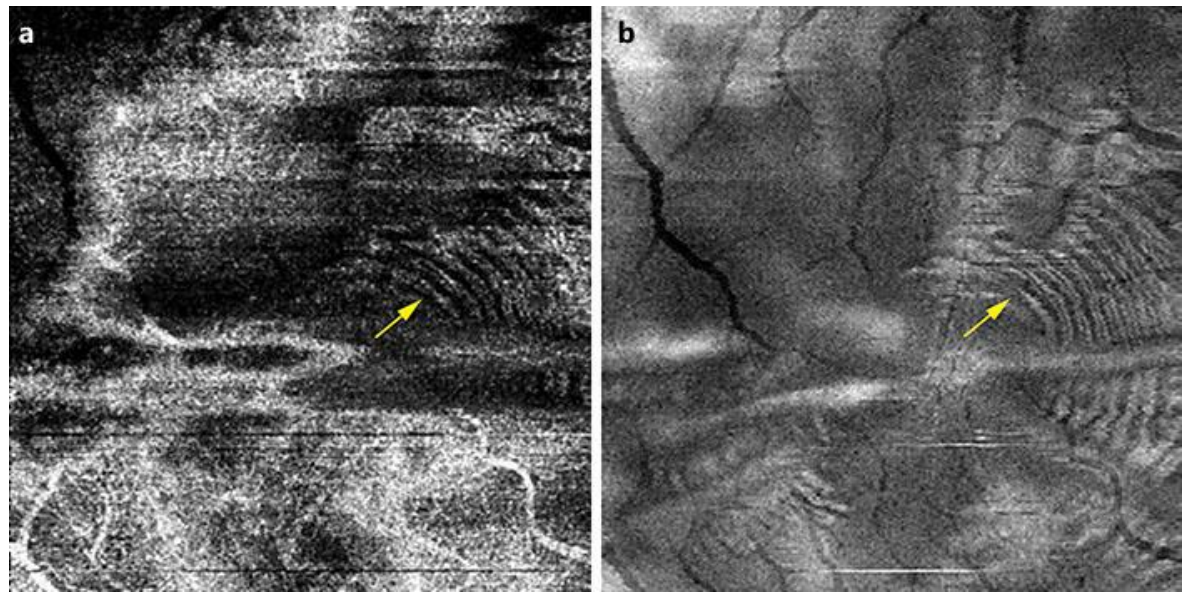

Fig. 4. A pattern of concentric cycles was noticed at the level of the deep capillary plexus corresponding to disruption of the outer plexiform layer due to intrusion of the outer nuclear layer. 